Open Access

\title{
Fundus autofluorescence in the diagnosis and monitoring of acute retinal necrosis
}

\author{
Tyson SJ Ward and Ashvini K Reddy*
}

\begin{abstract}
Background: Acute retinal necrosis (ARN), a vision threatening viral retinitis, is often diagnosed and treated based on clinical findings. These clinical features have been well characterized by various imaging modalities, but not using fundus autofluorescence (FAF), a noninvasive method of evaluating the neurosensory retina and retinal pigment epithelium (RPE) based on the detection of endogenous fluorophores.

Findings: A patient diagnosed with ARN was followed over a 10-month period to identify and document the fundus findings using FAF imaging. Pathological changes present at the level of the neurosensory retina and RPE in ARN can be detected and characterized using fundus autofluorescence imaging.

Conclusions: The borders of disease activity in ARN correlate with high-contrast changes in autofluorescence patterns to facilitate monitoring of disease progression.
\end{abstract}

Keywords: Acute retinal necrosis; Fundus autofluorescence

\section{Findings}

\section{Background}

Acute retinal necrosis (ARN) is a potentially blinding retinitis that was first reported in the 1970s [1,2]. It commonly occurs in immunocompetent individuals and is commonly associated with varicella zoster virus (VZV) and herpes simplex virus (HSV) [3]. The diagnosis of ARN is often made based on clinical features of anterior uveitis, vitritis, vasculitis, and peripheral areas of creamy white retinal necrosis. Secondary complications include rhegmatogenous retinal detachment, occlusive vasculopathy, neovascularization, vitreous hemorrhage, and phthisis bulbi [3].

The diagnostic clinical features of ARN previously described have been characterized by fundus photography, fluorescein angiography, optical coherence tomography [4], and electrophysiological studies [5].

In this report we describe variations in fundus autofluorescence (FAF) that were observed in a patient clinically diagnosed with ARN. FAF imaging is a noninvasive method of evaluating the neurosensory retina and retinal pigment epithelium (RPE) based on the fluorophores in these tissues [6]. Hyper-autofluorescence in FAF suggests

\footnotetext{
* Correspondence: reddy@virginia.edu

Department of Ophthalmology, University of Virginia, 1300 Jefferson Park Avenue, Charlottesville, VA 22901, USA
}

an increase in fluorophores of the RPE [7] or reduced blocking of fluorophores by damaged outer retina [8]. Decreased autofluorescence in FAF suggests atrophy or loss of RPE fluorophores [7].

\section{Method}

The patient was followed over 10 months with serial FAF images on both a Topcon 50EX retinal camera (Topcon, Paramus, NJ, USA) using an exciter filter of $585 \mathrm{~nm}$ and a barrier filter of $695 \mathrm{~nm}$ and a Spectralis HRA + OCT confocal scanning laser ophthalmoscope (Heidelberg Engineering, Heidelberg, Germany) using an excitation wavelength of $488 \mathrm{~nm}$. Although there is a difference in excitation wavelengths between the two FAF modalities used, this does not affect imaging results as comparison in the brightness of FAF images are not recommended even when using the same imaging modality. Relative comparisons can only be made within the same image with regard to intensity of hyperautofluoresence. A previous study showed that the green-light FAF images $(514 \mathrm{~nm})$ are superior for the accurate analysis of small, central, pathologic changes, and for the determination of the central geographical atrophy lesion size. Using only blue-light FAF could lead to an over interpretation of the size of atrophic patches and the center involvement [9]. Our literature search does 
not reveal any publication regarding this difference in ARN patients. This research was approved by the IRB at the University of Virginia.

\section{Case description}

A 64-year-old female with a history of mild leukopenia was referred with a 1 week history of progressively increasing floaters with 'fogging' of vision and photophobia in the left eye with no changes in the right eye. The patient reported a history of right-sided herpes zoster ophthalmicus 1 month prior for which she completed a 10 day course of famciclovir. Past medical history included chicken pox in preschool and genital herpes (diagnosed 22 years prior and treated with acyclovir). She denied a history of human immunodeficiency virus (HIV) disease.

Pinhole visual acuity was $20 / 25+1$ right eye (OD) and 20/60 left eye (OS). There were no vesicular lesions or preauricular lymphadenopathy on examination.
Slit lamp examination of the OD was unremarkable while the OS showed circumcorneal injection, mild anterior uveitis with moderate vitritis and haze. Fundus examination of the right eye was insignificant. Examination of the left eye was remarkable for vasculitis, a large area of wedge necrosis inferonasally from 6 to 7 o'clock and five small areas of necrosis temporally from approximately 3 to 5 o'clock with poorly defined margins (Figure 1).

The patient was clinically diagnosed with ARN and underwent anterior chamber paracentesis for viral polymerase chain reaction analysis, but there was insufficient fluid for analysis. She was admitted and started on IV acyclovir $600 \mathrm{mg}$ Q8 $\mathrm{h}$ for 7 days [3]. Laboratory bloodwork revealed elevated VZV IgG, HSV1 IgG and cytomegalovirus (CMV) IgG levels. On day 3, the patient agreed to $0.1 \mathrm{ml}$ Foscarnet $(2.4 \mathrm{mg} / 0.1 \mathrm{~mL})$ intravitreal injection OS $[10,11]$. On day 4 , the retinitis appeared

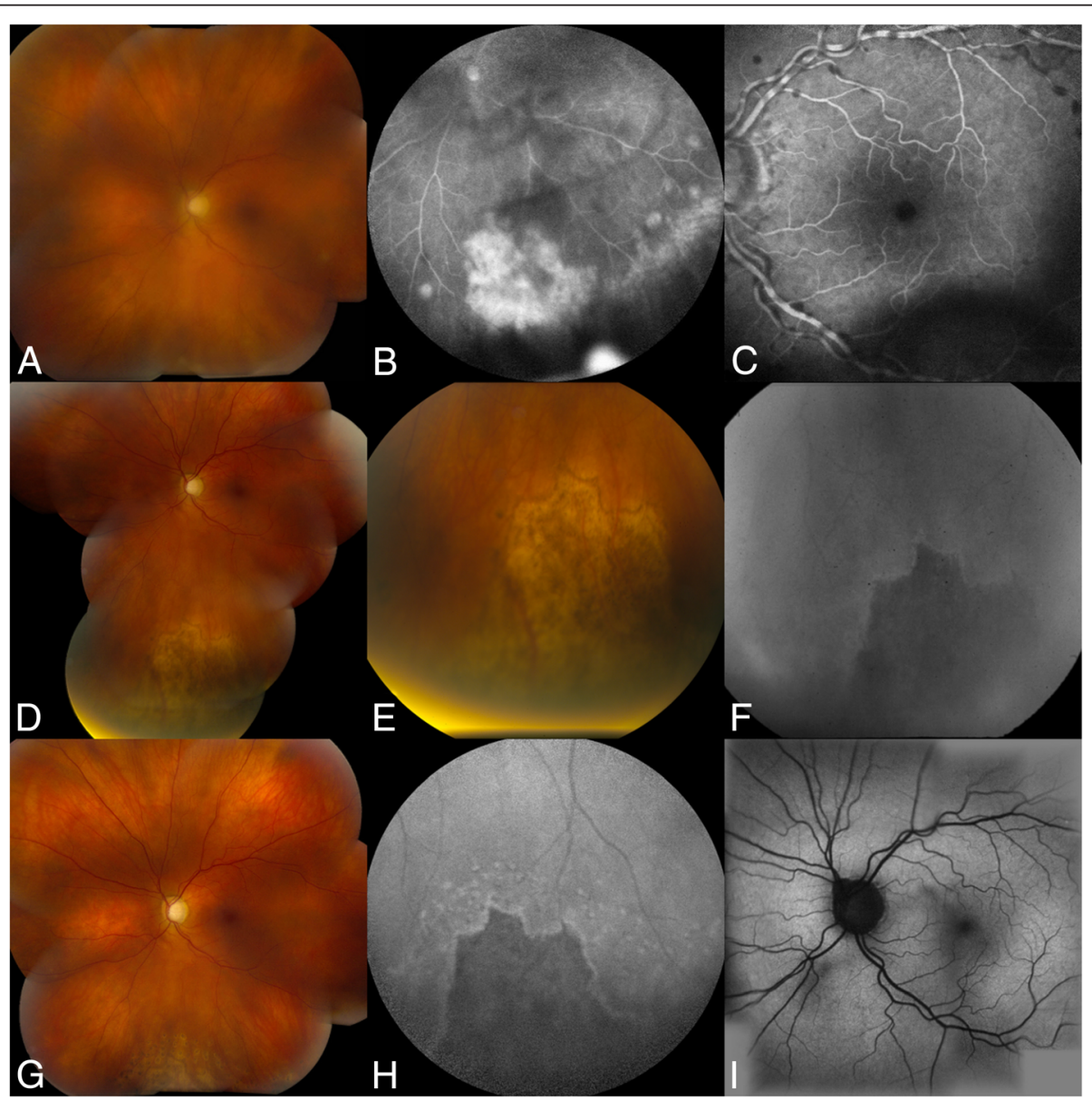

Figure $1 \mathrm{FAF}$ imaging results. (A-C) Color fundus photograph and fluorescein angiography demonstrating presentation of an ARN lesion and vasculitis. (D-F) Color fundus photographs and FAF imaging demonstrating hyperautofluorescent borders adjacent to areas of complete retinal necrosis characterized by hypoautofluorescence 3 months following presentation. (G-I) Five months after presentation and after photocoagulation treatment color photos and fundus autofluorescence images demonstrate arrest of the disease margin in high contrast. The lesion margins were stable (unchanged) at 10 months. 
improved and she was started on oral prednisone $60 \mathrm{mg}$ PO daily [3,12]. After completing a 7-day course of intravenous acyclovir, she was switched to valacyclovir $1,000 \mathrm{mg}$ PO TID and she steadily improved.

Over the course of the disease, the patient was followed with serial FAF images. At 1 month following presentation, ocular inflammation was markedly improved and the vision stabilized at 20/25 OS. No inflammatory activity was ever noted in the OD. The patient had laser barricade performed 3 months following her presentation $[3,13]$. She was slowly weaned off of oral steroids 4 months following presentation. She continues to be followed on a maintenance dose of valacyclovir 1,000 mg PO QD without evidence of disease reactivation.

\section{Results and discussion}

FAF changes have been reported in a case of PCRproven varicella zoster-associated progressive outer retinal necrosis (PORN) and herpes simplex virus retinitis $[13,14]$. In this patient with ARN, we report imaging characteristics using FAF.

Hyperautofluorescent borders surrounding areas of hypoautofluorescence corresponding to outer retinal damage adjacent to complete retinal necrosis and RPE atrophy can be appreciated [8] (Figure 1). The high contrast seen at the FAF borders of retinitis allowed for more precise determination of the extent and progression of the disease than the color photos. Furthermore, it is important to note that the hyperautofluorescent border by itself does not indicate active inflammation; it merely permits better contrast on FAF. If on repeated FAF imaging the hyperautofluoroscent borders are seen to be extending posteriorly, then it may imply spreading or active inflammation. Our patient had active inflammation at presentation but the disease was arrested with management, thus limiting the extension of hyperautofluoroscent borders on follow-up FAF. The areas of retinal atrophy seen on color fundus photography that previously demonstrated active retinitis on FA showed persistent hypoautofluorescence. The hyperautofluoroscent pattern seen at the borders in early photos does follow the same pattern as reported earlier in a case report of PORN [14]. However, these results do not corroborate previous findings published regarding FAF and posterior uveitis in which Reznicek et al. reported a larger area of involvement on FAF than color fundus images [15]. This could be explained because of photos being captured at different time frames or persistent active inflammation in the two HSV retinitis cases reported [15]. Larger case series of FAF imaging in ARN will provide further insights to its usefulness in management of the disease but is difficult given the rarity of the disease.

\section{Conclusion}

FAF permits visualization of a higher contrast border than color photos to help delineate lesions in ARN more accurately. The areas of retinal atrophy seen on color fundus photography that previously demonstrated active retinitis on FA showed persistent hypoautofluorescence. The borders of disease activity in ARN correlate with a high-contrast change in autofluorescence patterns that can be used to facilitate monitoring of disease progression.

\section{Consent}

The patient has consented for the report to be published.

\section{Abbreviations}

ARN: acute retinal necrosis; CMV: cytomegalovirus; FAF: fundus autofluorescence; HSV: herpes simplex virus; HIV: human immunodeficiency virus; PCR: polymerase chain reaction; PCR: retinal pigment epithelium; VZV: varicella zoster virus.

\section{Competing interests}

The authors declare that they have no competing interest.

\section{Authors' contributions}

AR provided clinical management for the patient including treatments and revising manuscript. TW assisted in clinical care of the patient and drafting the manuscript. Both authors read and approved the final manuscript.

Received: 15 October 2014 Accepted: 19 March 2015

Published online: 23 June 2015

\section{References}

1. Brown RM, Mendis U (1973) Retinal arteritis complicating herpes zoster ophthalmicus. Br J Ophthalmol 57:344-346

2. Young NJ, Bird AC (1978) Bilateral acute retinal necrosis. Br J Ophthalmol 62:581-590

3. Lau CH, Missotten T, Salzmann J, Lightman SL (2007) Acute retinal necrosis features, management, and outcomes. Ophthalmology 114:756-762

4. Suzuki J, Goto H, Minoda H, Iwasaki T, Sakai J, Usui M (2006) Analysis of retinal findings of acutre retinal necrosis using optical coherence tomography. Ocul Immunol Inflamm 14(3):165-170

5. Yamamoto S, Adachi-Usami E (1985) Electrophysiological studies on Kirisawa type uveitis (acute retinal necrosis). Doc Ophthalmol 60(1):93-100

6. Delori FC, Dorey CK, Staurenghi G, Arend O, Goger DG, Weiter JJ (1995) In vivo fluorescence of the ocular fundus exhibits retinal pigment epithelium lipofuscin characteristics. Invest Ophthalmol Vis Sci 36:718-729

7. Schmitz-Valckenberg S, Holz FG, Bird AC, Spaide RF (2008) Fundus autofluorescence imaging: review and perspectives. Retina 28:385-409

8. Freund KB, Mrejen S, Jung J, Yannuzzi LA, Boon CJ (2013) Increased fundus autofluorescence related to outer retinal disruption. JAMA Ophthalmol 131:1645-1649

9. Wolf-Schnurrbusch UE, Wittwer W, Ghanem R, Niederhaeuser M, Enzmann V, Framme C, Wolf S (2011) Blue-light versus green-light autofluorescence: lesion size of areas of geographic atrophy. Invest Ophthalmol Vis Sci 16:52(13):9497-9502

10. Luu KK, Scott IU, Chaudhry NA, Verm A, Davis JL (2000) Intravitreal antiviral injections as adjunctive therapy in the management of immunocompetent patients with necrotizing herpetic retinopathy. Am J Ophthalmol 129(6):811-813

11. Wong R, Pavesio CE, Laidlaw DA, Williamson TH, Graham EM, Stanford MR (2010) Acute retinal necrosis: the effects of intravitreal foscarnet and virus type on outcome. Ophthalmology 117(3):556-560

12. Barondes MJ, Tellez F, Siegel A (1992) Acute retinal necrosis after chickenpox in a healthy adult. Ann Ophthalmol 24:335-336

13. Han DP, Lewis H, Williams GA, Mieler W, Abrms GW, Aaberg TM (1987) Laser photocoagulation in the acute retinal necrosis syndrome. Arch Ophthalmol 105(8):1051-1054 
14. Yeh S, Wong WT, Weichel ED, Lew JC, Chew EY, Nussenblatt RB (2010) Fundus autofluorescence and optical coherence tomography in the management of progressive outer retinal necrosis. Ophthalmic Surg Lasers Imaging 9:1-4

15. Reznicek L, Seidensticker F, Stumpf C, Kampik A, Thurau S, Kernt M, Neubauer A (2013) Systematic analysis of wide-field fundus autofluorescence (FAF) imaging in posterior uveitis. Current eye research 39(2):164-171

Submit your manuscript to a SpringerOpen ${ }^{\odot}$ journal and benefit from:

- Convenient online submission

- Rigorous peer review

- Immediate publication on acceptance

- Open access: articles freely available online

- High visibility within the field

- Retaining the copyright to your article

Submit your next manuscript at $>$ springeropen.com 